\title{
Mitigating Global Cyber Risk Through Bridging the National Incident Response Capacity Gap
}

\author{
Elisabeth Dubois and Unal Tatar \\ University at Albany, State University of New York, Albany, NY, USA \\ evdubois@albany.edu \\ utatar@albany.edu
}

\begin{abstract}
Cyber-attacks know no borders. Given the globally connected environment, no region or country is secure against cyber-attacks unless the entire world is secure or has cyber capabilities. Yet, whether preventive or reactive, cyber countermeasures require coordination and engagement of various organizations, government bodies, and citizens of different countries. Although a variety of countermeasures exist, Computer Security Incident Response Teams (CSIRTs) have been deemed necessary systems in defending against and preventing cyberattacks, further supporting a nation's cyber capacity and limiting the harm to citizens, businesses, and governments. Despite calls for establishing CSIRTs at the national level, especially toward protecting critical infrastructure and lives from cyber threats, various discrepancies exist based on a nation's resources, capabilities, and needs. Limited research delves into the cyber capabilities of low-income countries despite an emphasis on improving global cyber capacity, leading to a need to establish a framework for low-income countries to address the unique needs with lessons learned from existing standards. CSIRTs can improve the cybersecurity posture of countries, so we seek to investigate how low-income countries can better mitigate cyber threats through cyber capacity building, including the creation of CSIRTs. This work-in-progress paper aims to investigate cyber incident response capacity building at the national level in low-income countries and identify challenges they may face in contributing to a more secure global cyberspace. Stemming from this paper, we will conduct a survey of national CSIRTs in low-income countries and conduct semi-structured interviews to further investigate their role. The implications of our research are far-spreading, assisting academics, practitioners, and governments in developing research, processes, and policies to aid low-income countries in their national cyber capacity building.
\end{abstract}

Keywords: cyber incident response, CSIRT, cybersecurity, capacity building, low-income country

\section{Introduction}

Cyber-attacks know no borders. In the digitally connected world where over 4.66 billion of the world, or about $60 \%$ of the globe, are digitally connected via the Internet (Johnson, 2021), no region or country is secure against cyber-attacks unless the entire world is secure or has cyber capabilities. Much like the effect globalization has had on the transmission of COVID-19, all nations are indeed connected, and thus no one nation is secure unless all are secure, at least to some extent. In 2020, cybercrimes resulted in a 1 trillion-dollar loss to the global government, which made up more than $1 \%$ of the global GDP and signaled a 50\% increase from 2018 (Smith et al., 2020). Worldwide, hundreds of thousands of cyber-attacks occur every day. Governments, businesses, critical infrastructure, and individuals face increased cyber risks, where cyber-physical risks to critical infrastructure are increasing exponentially. Think of the recent cyber-attack on Colonial Pipeline, which controls about half of the fuel supply along the East Coast of the United States. In fear that the attack had infected its systems, Colonial Pipeline turned them off, causing public panic with many buying at the gas pumps and spreading fear among the government that if not fixed soon, mass transit and factories would shut down as they would be unable to operate or distribute their products. Despite recovering, the ramifications of the pipeline attack are still being felt across the US. Concerns remain as to what will happen when a similar attack occurs in the US, other developed nations, or lower-income countries - will that country or the world be ready?

Most of the countermeasures to address cyber-attacks, whether preventive or reactive, require coordination and engagement of various organizations, government bodies, and citizens of different countries. At the forefront of these countermeasures are Computer Security Incident Response Teams (CSIRTs), which play a crucial role in national-level cyber security governance systems of countries, acting as the main coordinator and response team for cyber security incidents. CSIRTs can assume various other roles such as assisting in cyber security awareness and training, resolving incidents, cyber crisis planning and coordination activities, and/or recommending security notifications. In most countries, these teams initiate or promote the development of national-level policies and strategies. Thus, their roles often exceed basic incident coordination and awareness activities.

Globally, cybersecurity is viewed as not only a critical component to national and regional safety but a source of economic prosperity. National CSIRTs have been deemed active and necessary systems in defending against and 
preventing cyberattacks and cybercrimes by supporting a nation's cyber capacity and limiting the harm to citizens, businesses, and governments. Although there are universal calls for the establishment of CSIRTs at the national level, especially toward protecting critical infrastructure and lives from cyber threats, various discrepancies exist based on a nation's resources, capabilities, and needs. Despite many countries having established a national CSIRT, generally, low-income countries often are left behind or face unique challenges affecting their effectiveness in countering or protecting their nation from cyber threats.

Limited research delves into the cyber capabilities of low-income countries despite an emphasis on improving global cyber capacity. This signifies a need to establish a framework for low-income countries to address the unique needs of low-income countries and create solutions to better mitigate cyber-risks. Low-income countries are of particular interest in this study because besides the common challenges in CSIRT creation and operation other more developed nations may face, low-income countries face a host of factors that are compounded by their socioeconomic state. For this study, developing or low-income countries are defined due to 'their levels of per capita income' (Sanford and Sandhu, 2003). Low-income countries are those that have an annual per capita income between $\$ 875$ - $\$ 10,725$, still lack critical infrastructure investments, have lower living standards, an undeveloped industrial base, and low Human Development Index (HDI) (IGI Global, 2020).

This work-in-progress paper aims to begin exploring the challenges of cyber incident response capacity building in low-income countries and propose a study to identify policy implications and capacity-building solutions to result in secure global cyberspace. The implications of our proposed research are expansive, offering academics and practitioners a foundation to further investigate and build cyber-capacity. In finding a solution to the cyber capacity-building challenges in low-income countries, the world will be more secure from cyber risks.

This paper will outline what others have done in this space and emphasize CSIRT capacity building, identify some of the challenges of CSIRT creation, and present the next steps in conducting a survey and series of interviews with CSIRT members in low-income countries to evaluate the role, challenges, and best practices of capacity building.

\section{The Need for National CSIRTs}

Effective mechanisms and structures at the national level are necessary to deal with cyber threats and build capacity (ITU, 2021). The absence of such structures or lack of strong national capacities poses serious risks to nations and the world. Thus, national CSIRTs can provide a solution. According to the European Union Agency for Cybersecurity (ENISA), risk management needs to support organizational improvement, performance, and decision making (ENISA, 2021). Within the past 5 years, CSIRTs Network was created to build the capacity of the information systems in Europe (EU) through improved cyber capabilities at the national level, greater EU-level cooperation and risk management, and incident reporting obligations (ENISA, 2016). Within this, ENISA stipulates that CSIRTs are essential to capacity building and managing risks, where the new network will develop confidence and trust between the Member States and promote more timely and effective operational cooperation (2016). Likewise, the International Telecommunications Union highlights the importance of CSIRTs and how the establishment and development of CSIRTs should be based on mature models, utilizing international collaborations, strong procedures, effective tools, and trainings (ITU, 2021).

\section{Purpose of CSIRTs}

Utilizing CSIRTs for capacity building can be an effective way to mitigate risks. The FIRST'S CSIRT services framework classifies the services into five areas, including information security incident management, vulnerability management, information security event management, situational awareness, and knowledge transfer. These services are divided into reactive, proactive, and security quality management services (WestBrown et al., 2003). The services such as incident and vulnerability handling are presented as reactive. Proactive services range from intrusion detection to security assessments and maintenance of security infrastructures. The quality management service category covers the efforts related to training, awareness, consulting, risk analysis, and product evaluation.

Although CSIRTs work to improve many areas of cyber capacity, at the forefront of such capacity-building measures is the creation of a cybersecurity pipeline and human capabilities. The cyber security of any entity relies on the strength of its personnel and systems. As such, both developed and low-income countries should have CSIRT staff members that fulfill both the personal and technical skills to carry out cyber operations 
(Carnegie Mellon University, 2021). In this, national CSIRTs should have enough technical experts, as well as experts trained in communication. According to the recent publication of the Carnegie Mellon UniversitySoftware Engineering Institute (CMU-SEI), skills like communication, presentation, diplomacy, the ability to follow policies and procedures, teamwork, critical thinking, problem-solving, time management, integrity, and coping with stress are commonly referenced as beneficial skills for CSIRT members, while technical skills are grouped into two categories, technical foundation and incident handling skills (Carnegie Mellon University, 2021).

CSIRTs, in many ways, build off the global emphasis on personnel training and improving employee soft and technical skills. Besides gaining skill knowledge, CSIRTs stipulate that certificates or incident response courses are essential features for CSIRT members (Carnegie Mellon University, 2021). Practitioners and researchers emphasize how cyber incident response capabilities or CSIRTs can be strengthened through education and training. One major emphasis is on cybersecurity curricula in academic and professional settings and the need to incorporate industrial control system education and training to increase the effectiveness of incident response efforts of CSIRTs and government agencies (Marzitelli et al., 2015). Within this, CREST, an international not-forprofit organization (as well as a series of other organizations), helps professionals working towards qualifications or who would like to acquire, maintain, or extend their knowledge and build their capability (CREST, 2019).

Many CSIRTs offer lists of tools and processes aimed at helping build capacity or improve the effectiveness of CSIRTs. The National Cyber Security Centre in the Netherlands guide, as well as several other CSIRT guides, discusses the importance of appropriate tools and how the lack of sufficient tools can cause inefficient work and greater risk. Today, automated tools are one of the most critical weapons in a CSIRTs toolbox, as incident loads increase and cyber threats continue to grow (Van der Kleij et al., 2017).

Similarly, a host of national and international organizations and governments offer a variety of tools and trainings to assist in capacity building. In a literature review by Chowdhury \& Gkioulos (2021), they discuss that given the cyber threats to the national $\mathrm{Cl}$, various sectors have instilled trainings and testbeds to better preparedness against cyber threats. They found that cybersecurity training towards national security is split between awareness, technical, specialized CS, and incident response and recovery training (Chowdhury and Gkioulos, 2021). The Global Cyber Alliance (GCA) has developed several free cybersecurity tools and processes to meet the needs of CSIRTs and build cyber capacity. Within this, GCA provides several free toolkits to improve the cybersecurity and integrity of small businesses, elections, journalists, and financial institutions (Global Cyber Alliance, 2021). These toolkits vary in use and offerings, but they entail steps to not only improve the cyber hygiene of the particular party but provide tiered tools and recommendations for strong cyber protection across the board. Outside of these toolkits, GCA also offers a variety of useful tools to limit cyber risks via mobile and IoT devices and web searching, including Domain-based Message Authentication, Reporting, and Conformance (DMARC), Automated IoT Defence Ecosystem (AIDE), and Quad9. All of the tools provided by GCA are created in collaboration with other organizations, government entities, and individuals around the world to better address the challenges of cyber hygiene, response, and capacity building. Additionally, the Center for Internet Security publishes security guidelines, checklists, and tools for different platforms, operating systems, and other IT systems (Center for Internet Security, 2021). FIRST provides a list of resources for technical and procedural guidelines and hosts trainings on threat intelligence (FIRST, 2021). The International Telecommunications Union (ITU) also provides information about CSIRT tools as well as many procedural and training opportunities (International Telecommunication Union, 2021). Organizations and CSIRTs rely on SANS for free resources and tools to improve or build cyber security capabilities. The SANS Faculty Free Tools, alongside other resources, provide open source tools to support the work of organizations or governments to promote better security (SANS, 2021a). Within this, SANS also offers a number of quick reference guides and cheat sheets for IT security, digital forensics, incident response, offensive operations, cloud security, industrial control systems (ICS), and allaround defense (SANS, 2021b). A host of other tools are needed and utilized to store and track incidents, share cyber threat intelligence, coordinate data sharing and collaboration, as well as cater to one or more of the CSIRT services (including penetration testing, web application security).

\section{The Need for National CSIRTs in Low-Income Countries}

Although the need and purpose of national CSIRTs have been established, there are critical factors that make them necessary for low-income countries. As previously mentioned, low-income countries are those that have an annual per capita income between $\$ 875-\$ 10,725$, still lack critical infrastructure investments, have lower 
living standards, an undeveloped industrial base, and/or low Human Development Index (HDI) (IGI Global, 2020). Such countries include Nigeria, Indonesia, Bangladesh, Zambia, Cote d-Ivoire, Trinidad and Tobago, Togo, Malawi, etc.

At the forefront of the need for national CSIRTs among low-income countries is if low-income countries are not secure or do not have cyber capabilities, then the entire world faces greater risks. Yet, for low-income countries, more significant challenges exist in establishing a CSIRT. According to Grobler and Bryk, several challenges exist during the establishment of a CSIRT. Said challenges are clarifying mandates and policy issues, obtaining management support, securing funding, availability of staff and other resources, understanding cultural and governmental differences, training staff, and developing operational and technical procedures (Grobler and Bryk, 2010). Of these challenges CSIRT's ability to secure the relevant funds is vital. However, less-developed countries have fewer funding alternatives when compared to developed ones (Skierka and Hohmann, 2015). For these countries, some alternative options include government sponsorship, fee-based services, in-kind supports, or a combination of these could be funding alternatives for national CSIRTs (Jalal et al., 2018). Likewise, in lowincome countries, the availability of qualified staff or solid technology pipeline, training options, external support, and cultural differences create greater challenges due to limited critical infrastructure, lower living standards, underdeveloped industrial base, and low HDI. Through capacity-building support with international collaborations, affordable training opportunities, affordable tools for reliable infrastructure, and a greater focus on workforce improvement, low-income countries operating CSIRTs can better address the common challenges.

\section{Research Objectives}

This study will explore the challenges of cyber incident response capacity building at the national level for lowincome countries. The aims and objectives of this research are:

- Obj1: Identify the current CSIRT state in low-income countries (e.g., services delivered, personnel capacity, technical capability, technological infrastructure, legal base, and whenever applicable, identify the level of cybersecurity maturity for each of the countries, including governance structure)

- Obj2: Identify challenges of establishing and maintaining a national CSIRT

- Obj3: Identify solutions to mitigate the challenges of CSIRTs and enhance capacity building in lowincome countries.

\section{Next Steps: Methodology and Expected Findings}

Following an expanded desk review of academic and grey literature (e.g., reports of security vendors, independent organizations, government entities, national CSIRTs) to list the national CSIRT services and identify the relevant organizational models, applied incident handling processes, workflows, required human skill sets, training resources, applicable toolsets, and best practices in capacity development we plan to collect survey data and conduct interviews. A geographically diverse quantitative survey will be used to investigate the challenges of cyber incident response capacity building at the national level among low-income countries. We will aim for a representative sample of members of low-income countries CSIRTs or national cybersecurity teams. Following the survey, we plan to conduct select interviews with a handful of the survey respondents to identify challenges and best practices from a consultant's perspective and learn more about the available resources for low-income countries to develop CSIRT services.

Once completed, this study seeks to present solutions to mitigating cyber risks faced by low-income countries. Based on the desk review, such solutions may include capacity-building support through international collaborations, affordable training opportunities, affordable tools for reliable infrastructure, and a greater focus on workforce creation and a technology pipeline.

\section{References}

Carnegie Mellon University, 2021. Cyber Workforce Development [WWW Document]. Carnegie Mellon Univ. Softw. Eng. Inst. URL https://www.sei.cmu.edu/our-work/cyber-workforce-development/index.cfm (accessed 12.5.21).

Center for Internet Security, 2021. Center for Internet Security [WWW Document]. Cent. Internet Secur. URL https://www.cisecurity.org/ (accessed 12.5.21).

Chowdhury, N., Gkioulos, V., 2021. Cyber security training for critical infrastructure protection: A literature review. Comput. Sci. Rev. 40, 100361. https://doi.org/10.1016/j.cosrev.2021.100361

CREST, 2019. CREST Representing the Technical Information Security Industry.

ENISA, 2021. Risk Management [WWW Document]. Eur. Union Agency Cybersecurity ENISA. URL

https://www.enisa.europa.eu/topics/threat-risk-management/risk-management (accessed 12.5.21). 


\section{Elisabeth Dubois and Unal Tatar}

ENISA, 2016. CSIRTs Network [WWW Document]. Eur. Union Agency Cybersecurity ENISA. URL https://www.enisa.europa.eu/topics/csirts-in-europe/csirts-network (accessed 12.5.21).

FIRST, 2021. FIRST Security Reference Index [WWW Document]. FIRST — Forum Incid. Response Secur. Teams. URL https://www.first.org/resources/guides/reference (accessed 4.26.21).

Global Cyber Alliance, 2021. Global Cyber Alliance: Enabling a Secure and Trustworthy Internet [WWW Document]. GCA Glob. Cyber Alliance Work. Eradicate Cyber Risk. URL https://www.globalcyberalliance.org/ (accessed 3.31.21).

Grobler, M., Bryk, H., 2010. Common challenges faced during the establishment of a CSIRT, in: 2010 Information Security for South Africa. Presented at the 2010 Information Security for South Africa (ISSA), IEEE, Johannesburg, South Africa, pp. 1-6. https://doi.org/10.1109/ISSA.2010.5588307

IGI Global, 2020. What is Developing Countries | IGI Global [WWW Document]. URL https://www.igiglobal.com/dictionary/cyber-capability-framework/7401 (accessed 12.15.20).

International Telecommunication Union, 2021. ITU Cybersecurity Activities [WWW Document]. ITU. URL https://www.itu.int:443/en/action/cybersecurity/Pages/default.aspx (accessed 12.5.21).

ITU, 2021. National CIRT [WWW Document]. ITU. URL https://www.itu.int:443/en/ITU-D/Cybersecurity/Pages/nationalCIRT.aspx (accessed 12.5.21).

Jalal, I., Yusof, M., Shukur, Z., Mokhtar, M., 2018. A Model for Afghanistan's Cyber Security Incident Response Team. Int. J. Adv. Sci. Eng. Inf. Technol. 8, 2620-2626. https://doi.org/10.18517/ijaseit.8.6.6692

Johnson, J., 2021. Internet users in the world 2021 [WWW Document]. Statista. URL https://www.statista.com/statistics/617136/digital-population-worldwide/ (accessed 8.9.21).

Marzitelli, R., Monroe, K., Newmeyer, K., Parker, C., Pheils, D., Shih, L., Silas, M.A., Tu, M., 2015. National Cybersecurity Institute Journal 2, 80.

Sanford, J.E., Sandhu, A., 2003. Developing Countries: Definitions, Concepts and Comparisons. Nova Publishers.

SANS, 2021a. SANS Faculty Free Tools.

SANS, 2021b. The Ultimate List of SANS Cheat Sheets. Inst. URL https://www.sans.org/blog/the-ultimate-list-of-sans-cheatsheets/ (accessed 12.5.21).

Skierka, I., Hohmann, M., 2015. CSIRT Basics for Policy-Makers.

Smith, Z.M., Lostri, E., Lewis, J.A., 2020. The Hidden Costs of Cybercrime. McAfee.

Van der Kleij, R., Kleinhuis, G., Young, H., 2017. Computer Security Incident Response Team Effectiveness: A Needs Assessment. Front. Psychol. 8, 2179. https://doi.org/10.3389/fpsyg.2017.02179

West-Brown, M.J., Stikvoort, D., Kossakowski, K.-P., Killcrece, G., Ruefle, R., Zajicek, M., 2003. Handbook for Computer Security Incident Response Teams (CSIRTs) 223. 